\title{
Dynamics and interactions of spikes on smoothly curved boundaries for reaction-diffusion systems in 2D
}

\author{
Shin-Ichiro Ei • Toshio Ishimoto
}

Received: 11 October 2011 / Revised: 7 May 2012 / Published online: 29 July 2012

(C) The Author(s) 2012. This article is published with open access at Springerlink.com

\begin{abstract}
It is known that for special types of reaction-diffusion Systems, such as the Gierer-Meinhardt model and the Gray-Scott model, stable stationary spike solutions exist on boundary points with maximal curvature. In this paper, we rigorously give the equation describing the motion of spike solutions along boundaries for general types of reaction-diffusion systems in $\boldsymbol{R}^{2}$. We also apply the general results to the Gierer-Meinhardt model and show that a single spike solution moves toward a boundary point with locally maximal curvature. Moreover, by showing the repulsive interaction of spikes along boundaries for solutions of the Gierer-Meinhardt model, we have stable multispike stationary solutions in the neighborhood of a boundary point with locally maximal curvature.
\end{abstract}

Keywords Reaction-diffusion systems · Boundary spike solutions ·

The Gierer-Meinhardt model

Mathematics Subject Classification $\quad 35 \mathrm{~K} 61 \cdot 35 \mathrm{~K} 57$

\section{Introduction}

In 1972, Gierer and Meinhardt [9] proposed model equations to describe pattern formations in biology, according to the mechanism of diffusion-induced instability described by Turing [14]. The model equation is as follows:

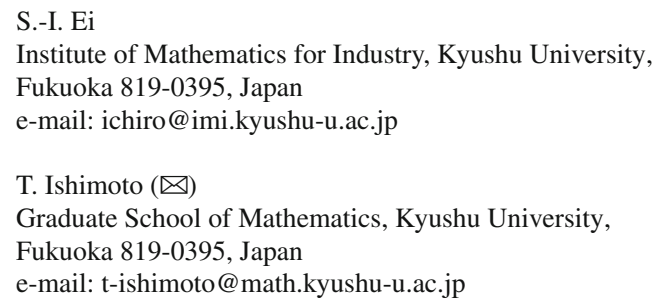




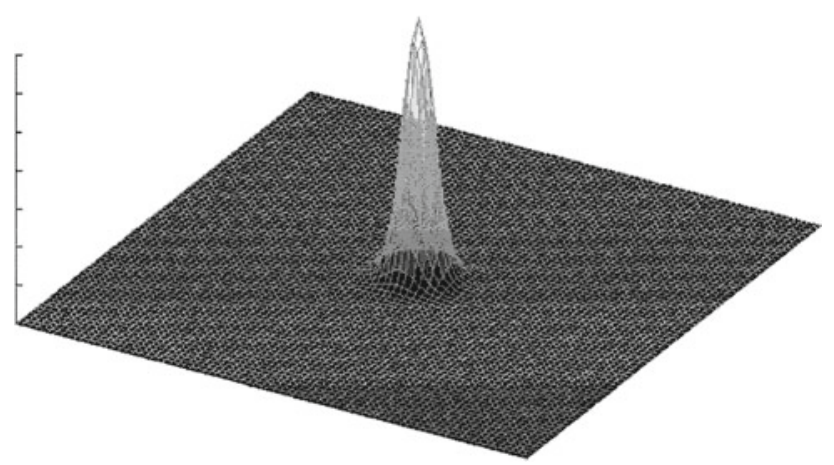

Fig. 1 Profile of a spike solution with one peak in $\boldsymbol{R}^{2}$. Only the $A$-component is drawn

$$
\left\{\begin{array}{l}
A_{t}=d_{1} \Delta A-A+\frac{A^{a_{1}}}{H^{a_{2}}}+a_{5}, \quad t>0, \boldsymbol{x} \in \Omega \subset \boldsymbol{R}^{n} \\
\tau H_{t}=d_{2} \Delta H-H+\frac{A^{a_{3}}}{H^{a_{4}}},
\end{array}\right.
$$

with the Neumann boundary conditions, where $d_{1}$ and $d_{2}$ are positive constants, and $a_{j} \geq 0$ are nonnegative constants satisfying

$$
0<\frac{a_{1}-1}{a_{2}}<\frac{a_{3}}{a_{4}+1}
$$

In the model, $d_{1}$ is assumed to be sufficiently small, and we write it as $d_{1}=\varepsilon^{2}$ for a sufficiently small $\varepsilon>0$.

One of the typical solutions of (1.1) is a spike solution. A spike solution has a profile such that the $A$-component is close to $A(\boldsymbol{x}) \sim \varepsilon^{-\eta} w(|\boldsymbol{x}| / \varepsilon)$ for $\eta>0$ and a radially symmetric function $w(r) \geq 0$. That is, the $A$-component has a sharp peak, as in Fig. 1. For the Gray-Scott model, similar spike solutions are observed [15,16].

There have been many works which studied the existence and stability of stationary spike solutions for (1.1) under appropriate conditions in one- or higher-dimensional spaces. We do not touch here on works related to one-dimensional problems of (1.1), because in this paper we consider (1.1) in two-dimensional spaces.

For higher-dimensional problems for (1.1), one of the most typical solutions is a boundary spike solution, which has peaks on the boundaries of its domain.

Related to boundary spike solutions in higher-dimensional spaces, a stationary spike solution with one peak on the boundary $\partial \Omega$ with globally maximum mean curvature was constructed in [13], and, later, a stationary spike solution with multiple peaks both inside $\Omega$ and on the boundary $\partial \Omega$ was constructed in [10]. Recently, [11] and [12] proved the existence and stability of a stationary spike solution with more than one peak at points with locally maximal mean curvatures of $\partial \Omega$ under the condition $a_{3}=a_{1}+1$ in (1.1). Thus, there has been much research and many results on stationary spike solutions with peaks on boundaries for (1.1), but we do not know a result on the dynamics of spikes along boundaries.

There have been several studies of the dynamics of solutions along boundaries for other models. Studies [1-5], dealt with the mass-conserving Allen-Cahn equations 
and/or the Cahn-Hilliard equations, and showed that small bubble solutions move along boundaries toward a boundary point with maximal mean curvature. This was proven using variational structures of systems together with other techniques.

On the other hand, many important examples of reaction-diffusion systems, such as the Gierer-Meinhard model and the Gray-Scott model, do not have such variational structures. But it is strongly expected that boundary spike solutions move toward points on boundaries with maximal mean curvature because many types of stable boundary spike solutions with peaks located at points with maximal mean curvature have been constructed.

In this paper, we give a general criteria necessary to study the dynamics and interactions of boundary spikes in a domain $\Omega \subset \boldsymbol{R}^{2}$, for general types of reaction-diffusion systems, and without assuming any variational structures. As one application of our results, we show the movement of a boundary spike solution for (1.1) toward a point on the boundary with locally maximal curvature. We also show the repulsive interaction between two boundary spikes and hence the existence of stable stationary solutions with two peaks in the neighborhood of a point with maximal curvature.

Here we note that there is a simplified version, called a shadow system, for the Gierer-Meinhardt model. It has a boundary spike solution, and a similar movement along the boundary is observed. But the treatment for the model is rather different from the one in this paper because the shadow system has nonlocal terms. This is reported in [7].

Now, we shall briefly discuss the results we present in this paper. Let $\Omega$ be a bounded domain in $\boldsymbol{R}^{2}$, and we assume that the boundary $\partial \Omega$ is a sufficiently smooth closed curve given by $\left\{\Gamma(s) \in \boldsymbol{R}^{2} ; 0 \leq s \leq s_{0}, \Gamma(0)=\Gamma\left(s_{0}\right)\right\}$, where $s$ is the arc-length parameter of $\partial \Omega$. Then we can take a tubular neighborhood of $\partial \Omega$ as $\boldsymbol{x}=(x, y)=\Gamma(s)+z v(s)$, where $v=v(s)$ is the inward normal unit vector of $\partial \Omega$ at $\Gamma(s)$. Define $\Sigma(\boldsymbol{x})$ and $Z(\boldsymbol{x})$ by the functions satisfying $\boldsymbol{x}=\Gamma(\Sigma(\boldsymbol{x}))+Z(\boldsymbol{x}) \nu(\Sigma(\boldsymbol{x}))$, and let $\kappa=\kappa(s)$ be the curvature of $\partial \Omega$ at $\Gamma(s)$ measured in the direction of $\nu$. We consider general types of reaction-diffusion systems:

$$
U_{t}=\delta^{2} D \Delta U+F(U), \quad t>0, x \in \Omega
$$

with the Neumann boundary condition. Here, $U=U(t, \boldsymbol{x}) \in \boldsymbol{R}^{N}, D:=\operatorname{diag}\left(d_{1}\right.$, $\left.d_{2}, \cdots, d_{N}\right)$ for $d_{j}>0$, and $\delta>0$ is a sufficiently small constant. Let $\mathcal{L}_{\delta}(U):=$ $\delta^{2} D \Delta U+F(U)$.

First we consider

$$
U_{t}=D \Delta U+F(U), \quad t>0, \zeta \in \boldsymbol{R}_{+}^{2}
$$

with the Neumann boundary condition, where $\boldsymbol{R}_{+}^{2}:=\left\{\boldsymbol{\zeta}:=(l, \mu) \in \boldsymbol{R}^{2}\right.$; $-\infty<l<\infty, \mu>0\}$. Let $\mathbf{0}:=(0, \cdots, 0) \in \boldsymbol{R}^{N}$. We assume as follows:

(H1) $F(\mathbf{0})=\mathbf{0}$ is satisfied and (1.3) has a stationary radially symmetric solution, say $S=S(r)$, on the boundary $\partial \boldsymbol{R}_{+}^{2}$ with asymptotic form $S(r) \rightarrow \frac{1}{\sqrt{r}} e^{-\alpha r} \boldsymbol{a}$, where $r=\sqrt{l^{2}+\mu^{2}}, \alpha>0$ and $\boldsymbol{a} \in \boldsymbol{R}^{N}$. 
Let $\mathcal{A}(U):=D \Delta U+F(U), A:=\mathcal{A}^{\prime}(S(r))$, and $A_{0}:=\mathcal{A}^{\prime}(\mathbf{0})$ in $\boldsymbol{R}_{+}^{2}$. We assume the stability of $\mathbf{0}$ and $S$ for (1.3).

(H2) $S(r)$ is stable in the linearized sense, that is, the spectral set $I(A)$ of $A$ is $I(A) \subset$ $I_{0} \cup I_{1}$, where $I_{0}:=\{0\}$ and $I_{1} \subset\left\{\operatorname{Re} \lambda<-\gamma_{0}\right\}$ for $\gamma_{0}>0$, and 0 is simple.

(H2)' $\mathbf{0}$ is a stable equilibrium for (1.3) in the linearized sense. That is, the spectral set $I\left(A_{0}\right)$ of $A_{0}$ satisfies $I\left(A_{0}\right) \subset\left\{\operatorname{Re} \lambda<-\gamma_{0}\right\}$.

Note that $A \partial_{l} S=0$. Hereinafter we denote $\partial_{l} S(r(l, \mu))$ simply by $\partial_{l} S$ for $r=\sqrt{l^{2}+\mu^{2}}$. Other cases, such as when $r$ is a different function, are treated similarly, while we denote $\frac{d S}{d r}$ by $S_{r}$. Let $A^{*}$ be the adjoint operator of $A$, and $\phi^{*}(\boldsymbol{x})$ be the eigenfunction satisfying $A^{*} \phi^{*}=0$ with $\left\langle\partial_{l} S, \phi^{*}\right\rangle_{2}=\frac{\pi}{2}$, where $\langle U, V\rangle_{2}:=\int_{\boldsymbol{R}_{+}^{2}}\langle U, V\rangle d \zeta$. We note that $\partial_{l} S=\cos \theta S_{r}=\frac{l}{r} S_{r}$ and that $\phi^{*}$ is also given by $\phi^{*}(\zeta)=\partial_{l} \Phi^{*}=\cos \theta \Phi_{r}^{*}=\frac{l}{r} \Phi_{r}^{*}$ for a radially symmetric function $\Phi^{*}(r)$ under suitable conditions, where $l=r \cos \theta$ and $\mu=r \sin \theta$, which will be mentioned in the next section. Then the normalization $\left\langle\partial_{l} S, \phi^{*}\right\rangle_{2}=\frac{\pi}{2}$ implies that $\left\langle S_{r}, \Phi_{r}^{*}\right\rangle_{R}=1$, where $\langle U, V\rangle_{R}:=\int_{0}^{\infty} r\langle U, V\rangle d r$ for radially symmetric functions $U=U(r)$ and $V=V(r) \in \boldsymbol{R}^{N}$.

Now, coming back to the original problem (1.2) in $\Omega$, we show the following: Let $r(\boldsymbol{x}, h):=\sqrt{(\Sigma(\boldsymbol{x})-h)^{2}+Z^{2}(\boldsymbol{x})},\left(0 \leq h \leq s_{0}\right)$, and

$$
M_{0}:=-\frac{8}{15 \pi}\left(2 \int_{0}^{\infty} r^{2}\left\langle D S_{r}, \Phi_{r}^{*}\right\rangle d r+3 \int_{0}^{\infty} r^{3}\left\langle D S_{r r}, \Phi_{r}^{*}\right\rangle d r\right) .
$$

Then the solution $U(t, \boldsymbol{x})$ of (1.2) keeps close to $S(r(\boldsymbol{x}, h(t)) / \delta)$, and $h(t)$ is governed by the dynamics

$$
\dot{h}=M_{0} \delta^{3} \frac{d \kappa}{d s}(h)+O\left(\delta^{4}\right),
$$

where $\dot{h}$ denotes $\frac{d h}{d t}$. These results are proved by using invariant manifold theory (e.g. [5]), while we need to obtain explicit estimates of several objects, such as spectrum and resolvent, for (1.2).

The above results are applied to the Gierer-Meinhardt model of the form

$$
\left\{\begin{array}{l}
u_{t}=\varepsilon^{2} \Delta u-u+\frac{u^{a_{1}}}{(v+a)^{a_{2}}}, \\
\tau v_{t}=d \Delta v-v+\frac{u^{a_{3}}}{(v+a)^{a_{4}}}
\end{array}\right.
$$

where $\tau$ and $a$ are nonnegative constants, and the $a_{j}$ are the same ones as (1.1). If both $\tau$ and $a$ are positive or $\tau=a=0$ in (1.5), the case has been treated on the whole $\boldsymbol{R}^{2}$ space in [8], and, by using the results, the constant $M_{0}$ is shown to be positive. This shows that $h(t)$ approaches the point of the maximal $\kappa(s)$, that is, the point with the maximal curvature of $\partial \Omega$ (Fig. 2).

Thus, our results give the well-known results for a stable boundary spike solution of the Gierer-Meinhardt model $[9,11-13,16]$ from the viewpoint of dynamics. 


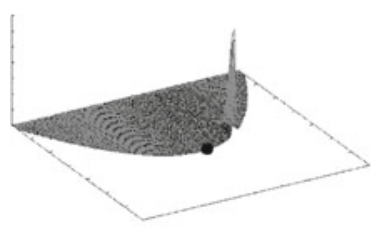

(a) $t=0$

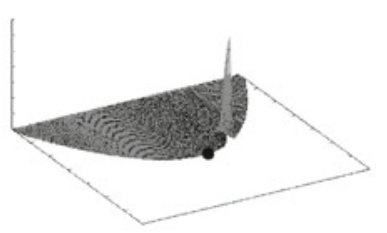

(b) $t=100$

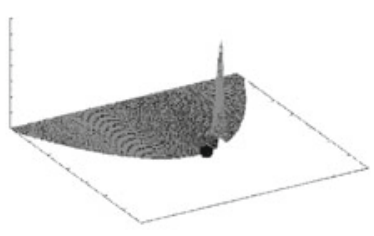

(c) $t=300$

Fig. 2 Movement of a boundary spike toward a point with maximal curvature, which is denoted by a black circle in the figure. The parameter values are $\tau=0.5, \varepsilon=0.01, d=1.0, a=0.01, a_{1}=2$, $a_{2}=2, a_{3}=3$, and $a_{4}=3$

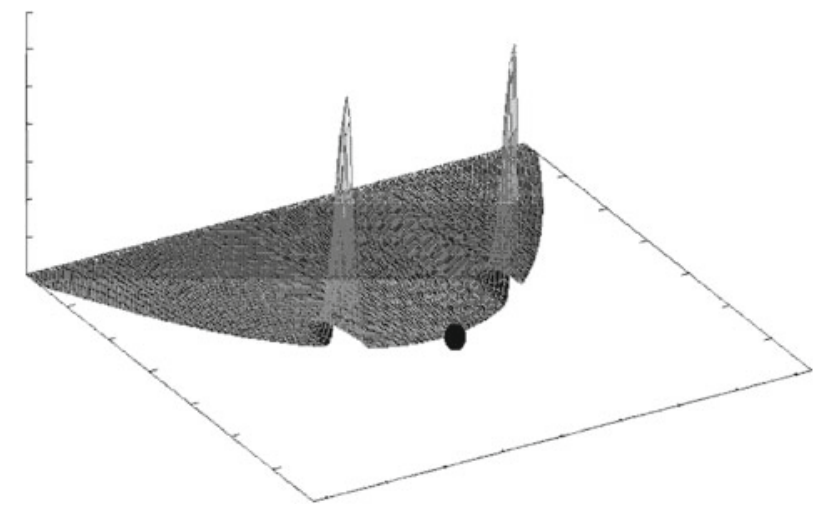

Fig. 3 Stable stationary solution with two peaks in the neighborhood of a point with maximal curvature, which is denoted by a black circle in the figure. The parameter values are the same as in Fig. 2

We can also show the existence of a stable stationary solution with two peaks in the neighborhood of a point with maximal curvature of $\partial \Omega$ by using the repulsive interaction (Fig. 3). The idea was presented in [8].

In this paper, only the two-dimensional case is considered. All the arguments and techniques can be applied to the higher dimensional cases, too but we do not do it here.

\section{Main results}

Define $\Omega\left(z_{0}\right):=\left\{\boldsymbol{x}=\Gamma(s)+z v(s), 0 \leq s \leq s_{0}, 0 \leq z<z_{0}\right\}$, where we regard $\Gamma(s)$ and $v(s)$ as periodic functions of $s$ with period $s_{0}$. We fix $N_{1}>0$ and represent $\Omega=$ $\Omega_{0} \cup \Omega_{1}$, where $\Omega_{1}:=\Omega\left(2 N_{1} \sqrt{\delta}\right)$ and $\Omega_{0}:=\Omega \backslash \Omega\left(N_{1} \sqrt{\delta}\right)$. Hereinafter, $c, c_{j}, c_{j}^{\prime}$, and $\gamma_{j}$ denote general positive constants independent of $\delta$. Let $\chi_{0}(\boldsymbol{x})$ and $\chi_{1}(\boldsymbol{x})$ be cutoff functions such that $0 \leq \chi_{j}(\boldsymbol{x}) \leq 1, \chi_{0}(\boldsymbol{x})+\chi_{1}(\boldsymbol{x})=1, \chi_{0}(\boldsymbol{x})=1$, and $\chi_{1}(\boldsymbol{x})=0$ for $\boldsymbol{x} \in \Omega \backslash \Omega_{1}$; and $\chi_{0}(\boldsymbol{x})=0$ and $\chi_{1}(\boldsymbol{x})=1$ for $\boldsymbol{x} \in \Omega \backslash \Omega_{0}=\Omega\left(N_{1} \sqrt{\delta}\right)$.

In the tubular neighborhood $\Omega_{1}$, define the coordinates $s=\Sigma(\boldsymbol{x})$ and $z=Z(\boldsymbol{x})$ by $\boldsymbol{x}=\Gamma(s)+z v(s)$. Define $S(\boldsymbol{x} ; h):=\chi_{1}(\boldsymbol{x}) S(r(\boldsymbol{x} ; h) / \delta)$, where $r(\boldsymbol{x} ; h):=$ $\sqrt{(\Sigma(\boldsymbol{x})-h)^{2}+Z(\boldsymbol{x})^{2}}$. Here, we extend $r(\boldsymbol{x} ; h)$ to the whole domain $\Omega$ so as to satisfy $c_{1}^{\prime}|\boldsymbol{x}-\Gamma(h)| \leq r(\boldsymbol{x} ; h) \leq c_{2}^{\prime}|\boldsymbol{x}-\Gamma(h)|$ uniformly for $\boldsymbol{x} \in \Omega$ and $0 \leq h \leq s_{0}$. 
Let $\Omega_{2}:=\Omega_{0} \cap \Omega_{1}$. Since $S(r)$ satisfies $S(r) \rightarrow \frac{1}{\sqrt{r}} e^{-\alpha r} \boldsymbol{a}$ as $r \rightarrow+\infty, S(\boldsymbol{x} ; h) \leq$ $O\left(e^{-c / \sqrt{\delta}}\right)$ in $\Omega_{2}$.

We add the following assumption. Let $X_{R}:=\left\{U=U(r) ;\|U\|_{R}^{2}:=\langle U, U\rangle_{R}<\right.$ $\infty\}$ be a set of the radially symmetric functions, and define $A_{R} U:=D\left(U_{r r}+\frac{1}{r} U_{r}\right)+$ $F^{\prime}(S(r)) U, \widehat{A}_{R} U:=D\left(U_{r}+\frac{1}{r} U\right)_{r}+F^{\prime}(S(r)) U$. Note that $A_{R}$ is the restriction of $A$ in $X_{R}$, and $\widehat{A}_{R} S_{r}=0$. Then we assume:

(H3) 0 is an isolated simple eigenvalue of $\widehat{A}_{R}$, and $A_{R}$ is invertible in $X_{R}$.

If (H3) holds, there exists $\phi_{0}^{*}(r) \in X_{R}$ such that $\widehat{A}_{R}^{*} \phi_{0}^{*}=0$ and $\left\langle S_{r}, \phi_{0}^{*}\right\rangle_{R}=1$, where $\widehat{A}_{R}^{*}$ is the adjoint operator of $\widehat{A}_{R}$. Then setting $\Phi^{*}(r):=\int^{r} \phi_{0}^{*}(r) d r$, we have $\phi^{*}(\zeta)=\partial_{l} \Phi^{*}(r)=\cos \theta \Phi_{r}^{*}(r)=\cos \theta \phi_{0}^{*}(r)=\frac{l}{r} \phi_{0}^{*}(r)$ for $r=\sqrt{l^{2}+\mu^{2}}$, and the normalization $\left\langle S_{r}, \phi_{0}^{*}\right\rangle_{R}=1$ implies $\left\langle\partial_{l} S, \phi^{*}\right\rangle_{2}=\frac{\pi}{2}$ because $A^{*} \phi^{*}=\cos \theta \widehat{A}_{R}^{*} \phi_{0}^{*}=0$.

Theorem 2.1 Assume $(\mathrm{H} 1)$ to $(\mathrm{H} 3)$ and that the initial data $U_{0}(\boldsymbol{x})$ is sufficiently close to $S\left(\boldsymbol{x} ; h_{0}\right)$ for $0 \leq h_{0} \leq s_{0}$. Then for a positive constant $c$ the solution $U(t, \boldsymbol{x})$ of (1.2) and $h(t)$ satisfy

$$
\|U(t, \cdot)-S(\cdot ; h(t))\|_{\infty} \leq c \delta
$$

and (1.4) uniformly for any $t>0$ and sufficiently small $\delta>0$.

Let us consider the reduced ODE of (1.4)

$$
\dot{h}=M_{0} \delta^{3} \frac{d \kappa}{d s}(h) .
$$

Corollary 2.1 If $h^{*}$ is a stable (or unstable) equilibrium of (2.1) in the linearized sense, that is $M_{0} \frac{d^{2} \kappa}{d s^{2}}\left(h^{*}\right)<0$ (or $\left.>0\right)$, then there exists a stable (or unstable) stationary solution $U^{*}(\boldsymbol{x})$ of $(1.2)$ satisfying $\left\|U^{*}(\cdot)-S\left(\cdot ; h^{*}\right)\right\|_{\infty} \leq c \delta$ for $c>0$.

Next we give the results for the movement of multispike solutions on the boundary.

Define $G(r):=\int_{r}^{\infty}\left\langle F^{\prime}(S(r))-F^{\prime}(\mathbf{0}) \boldsymbol{a}, \phi^{*}(r)\right| d r$ and $M_{1}:=\int_{0}^{\infty} r G(r) d r$ $\int_{0}^{\pi} e^{\sin \theta} d \theta$.

Theorem 2.2 Suppose the initial data $U_{0}(\boldsymbol{x})$ is sufficiently close to $S\left(\boldsymbol{x} ; h_{1}\right)+$ $S\left(\boldsymbol{x} ; h_{2}\right)$ for $c_{1}<h_{1}<h_{2}<s_{0}-c_{1}$ with $c_{2}|\delta \log \delta|<h_{2}-h_{1}<\frac{1}{2} c_{1}$ for positive constants $c_{1}$ and $c_{2}$. Then

$$
\left\{\begin{array}{l}
\dot{h_{1}}=M_{0} \delta^{3} \frac{d \kappa}{d s}\left(h_{1}\right)-M_{1} \sqrt{\frac{\delta}{h}} e^{-\alpha h / \delta}\left(1+O\left(\frac{\delta}{h}\right)\right)+O\left(\delta^{4}\right), \\
\dot{h_{2}}=M_{0} \delta^{3} \frac{d \kappa}{d s}\left(h_{2}\right)+M_{1} \sqrt{\frac{\delta}{h}} e^{-\alpha h / \delta}\left(1+O\left(\frac{\delta}{h}\right)\right)+O\left(\delta^{4}\right)
\end{array}\right.
$$

as long as $c_{1}<h_{1}<h_{2}<s_{0}-c_{1}$ with $c_{2}|\delta \log \delta|<h_{2}-h_{1}<\frac{1}{2} c_{1}$, where $h:=h_{2}-h_{1}$. 
Remark 2.1 The restriction of the range of $h_{1}$ and $h_{2}$ is not necessary because we may consider $h_{1}$ and $h_{2}$ with $\bmod s_{0}$. However, the statement of Theorem 2.2 then becomes complicated, and so, for simplicity, we restrict the range in the theorem.

Consider the reduced system of (2.2)

$$
\left\{\begin{array}{l}
\dot{h_{1}}=M_{0} \delta^{3} \frac{d \kappa}{d s}\left(h_{1}\right)-M_{1} \sqrt{\frac{\delta}{h}} e^{-\alpha h / \delta}, \\
\dot{h_{2}}=M_{0} \delta^{3} \frac{d \kappa}{d s}\left(h_{2}\right)+M_{1} \sqrt{\frac{\delta}{h}} e^{-\alpha h / \delta} .
\end{array}\right.
$$

Suppose that $M_{0}, M_{1}$ are positive and that $h^{*}$ is a stable equilibrium of (2.1) in the linearized sense. Then we can check that (2.3) has a linearly stable equilibrium, say $H^{*}=\left(h_{1}^{*}, h_{2}^{*}\right)$ with $h_{1}^{*}<h^{*}<h_{2}^{*}$ and $h_{2}^{*}-h_{1}^{*}=O(|\delta \log \delta|)$. The proof is given in Sect. 6.

Corollary 2.2 Under the above assumptions, there exists a stable stationary solution $U^{*}(\boldsymbol{x})$ of $(1.2)$ satisfying $\left\|U^{*}(\cdot)-\left\{S\left(\boldsymbol{x} ; h_{1}^{*}\right)+S\left(\boldsymbol{x} ; h_{2}^{*}\right)\right\}\right\|_{\infty} \leq c \delta$ for $c>0$.

$U^{*}(\boldsymbol{x})$ has a profile with two peaks in the neighborhood of a boundary point with maximal curvature.

Corollary 2.3 For (1.5), suppose that both $\tau$ and $a$ are positive, or both $\tau$ and a are equal to zero. If $a_{3}=2$ and $1<a_{1}<3$, or $a_{3}=a_{1}+1$ for $1<a_{1}<\infty$, then the constant $M_{0}$ is positive. That is, a spike on the boundary moves toward a point with maximal curvature. Moreover, $M_{1}$ is also positive, which implies the existence of a stable stationary solution of (1.5) with two peaks in the neighborhood of a point with maximal curvature, as stated in Corollary 2.2.

\section{Formal derivation of (1.4)}

In this section, we formally derive the ordinary differential equation (ODE) (1.4). Equation (1.2) is represented by

$$
U_{t}-\frac{h_{t}}{\delta} U_{l}=D\left\{U_{\mu \mu}-\frac{\delta \kappa}{1-\delta \mu \kappa} U_{\mu}+\frac{1}{1-\delta \mu \kappa}\left(\frac{1}{1-\delta \mu \kappa} U_{l}\right)_{l}\right\}+F(U)
$$

for $\boldsymbol{x} \in \Omega_{1}$ if we take $U(t, \boldsymbol{x})=U(t,(s-h(t)) / \delta, z / \delta)$ with $s=\Sigma(\boldsymbol{x}), z=$ $Z(\boldsymbol{x}), \delta l:=s-h(t)$, and $\delta \mu:=z$. Since $\delta$ is sufficiently small, we can regard $\Omega_{1}$ as approximately $\boldsymbol{R}_{+}^{2}$. Let

$$
K^{\delta}(l, \mu) U:=\frac{1}{\delta} D\left\{-\frac{\delta \kappa}{1-\delta \mu \kappa} U_{\mu}+\frac{1}{1-\delta \mu \kappa}\left(\frac{1}{1-\delta \mu \kappa} U_{l}\right)_{l}-U_{l l}\right\} .
$$

Although $K^{\delta}$ is defined only in $\Omega_{1}$, we may assume it is appropriately extended in $\boldsymbol{R}_{+}^{2}$, e.g. by multiplying $K^{\delta}$ by cut-off functions disappearing outside of $\Omega_{1}$ and for sufficiently large $\mu$. Then (3.1) is written as 


$$
U_{t}-\frac{h_{t}}{\delta} U_{l}=D \Delta_{l, \mu} U+F(U)+\delta K^{\delta}(l, \mu) U, \quad t>0,(l, z) \in \boldsymbol{R}_{+}^{2} .
$$

Substituting $U(t, l, \mu)=S(r)+V(t, l, \mu)$ into (3.2) for $V \in E^{\perp}$, we have

$$
V_{t}-\frac{h_{t}}{\delta}\left(\cos \theta S_{r}+V_{l}\right)=A V+\delta K^{\delta} S+O\left(|V|^{2}+\delta^{2}\right),
$$

where $l=r \cos \theta, \mu=r \sin \theta$, and $E^{\perp}:=\left\{V ;\left\langle V, \phi^{*}\right\rangle_{2}=0\right\}$. Now we assume $V=V(T, l, \mu)$ with $T:=\delta t$, and may set $h_{t}=\delta h_{T}=\delta\left(H_{0}+\delta H_{1}+\delta^{2} H_{2}+O\left(\delta^{3}\right)\right)$ and $V=\delta V_{1}+\delta^{2} V_{2}+O\left(\delta^{3}\right)$.

Since $\kappa=\kappa(h(t)+\delta l)=\kappa(h(t))+\delta l \kappa_{s}(h(t))+O\left(\delta^{2}\right), K^{\delta}$ is expanded as $K^{\delta}=K_{1}+\delta K_{2}+O\left(\delta^{2}\right)$, where

$$
\begin{aligned}
K_{1} U:= & \kappa(h) D\left(-U_{\mu}+2 \mu U_{l l}\right), K_{2} U:=\kappa_{s}(h) D\left(\mu U_{l}-l U_{\mu}+2 \mu l U_{l l}\right) \\
& +\kappa^{2}(h) D\left\{-\mu U_{\mu}+3 \mu^{2} U_{l l}\right\} .
\end{aligned}
$$

$H_{0}=0$ is easily shown. Considering terms of order $\delta$, we have

$$
-H_{1} \cos \theta S_{r}=A V_{1}+K_{1} S \text {. }
$$

Taking the inner product of (3.4) with $\phi^{*}(l, \mu)=\cos \theta \Phi_{r}^{*}(r)$, we have

$$
-\frac{\pi}{2} H_{1}=\left\langle K_{1} S, \phi^{*}\right\rangle_{2}
$$

Lemma 3.1 $H_{1}=0$.

Proof Since $\partial_{\mu} S=\sin \theta S_{r}$ and $\partial_{l}^{2} S=\frac{\sin ^{2} \theta}{r} S_{r}+\cos ^{2} \theta S_{r r}$, the direct calculation of the right-hand side of (3.5) gives $H_{1}=0$.

In order to obtain $\mathrm{H}_{2}$, we now consider terms of order $\delta^{2}$ of (3.3). Then we have

$$
\partial_{T} V_{1}-H_{2} \cos \theta S_{r}=A V_{2}+K_{2} S+K_{1} V_{1}+\frac{1}{2} F^{\prime \prime}(S) V_{1}^{2} .
$$

First, we note that $\partial_{T} V_{1}=O\left(h_{T}\right)=O(\delta)$ because $H_{1}=0$. Hence from (3.6), $H_{2}$ is given by

$$
-\frac{\pi}{2} H_{2}=\left\langle K_{2} S+K_{1} V_{1}+\frac{1}{2} F^{\prime \prime}(S) V_{1}^{2}, \phi^{*}\right\rangle_{2} .
$$

Since $V_{1} \in E^{\perp}$ is a unique solution of $0=A V_{1}+K_{1} S$ and $K_{1} S$ is even with respect to $l, V_{1}=V_{1}(l, z)$ is also even with respect to $l$. Hence $\left\langle K_{1} V_{1}, \phi^{*}\right\rangle_{2}$ $=\left\langle\frac{1}{2} F^{\prime \prime}(S) V_{1}^{2}, \phi^{*}\right\rangle_{2}=0$ and

$$
-\frac{\pi}{2} H_{2}=\left\langle K_{2} S, \phi^{*}\right\rangle_{2}
$$


hold in (3.7). The right-hand side is directly calculated as

$$
\left\langle K_{2} S, \phi^{*}\right\rangle_{2}=\left\langle K_{2} S, \cos \theta \Phi_{r}^{*}\right\rangle_{2}=-\frac{4}{15} M_{0} \kappa_{S}(h),
$$

and we have $H_{2}=\frac{8}{15 \pi} M_{0} \kappa_{s}(h)$. This shows (1.4).

\section{Proofs of Theorem 2.1 and Corollary 2.1}

\subsection{Proof of Theorem 2.1}

Let $L(h):=\mathcal{L}_{\delta}^{\prime}(S(\boldsymbol{x} ; h))=\delta^{2} D \Delta+F^{\prime}(S(\boldsymbol{x} ; h))$ in $\Omega$, and $X:=C_{\text {unif }}(\Omega)$ with the sup-norm $\|\cdot\|$.

Lemma 4.1 The spectral set, say $I(h)$ of $L(h)$, is given by $I(h)=I_{0}(h) \cup I_{1}(h)$, where $I_{0}(h) \subset\{|\lambda| \leq c \sqrt[4]{\delta}\}$ and $I_{1}(h) \subset\left\{\right.$ Re $\left.\lambda<-\gamma_{1}\right\}$ for positive constants $c$ and $\gamma_{1}$.

Proof We take $0<\gamma_{1}<\gamma_{0}$ such that $\left\{\operatorname{Re} \lambda>-\gamma_{1}\right\} \subset \rho\left(A_{0}\right)$, the resolvent set of $A_{0}$.

First, we consider $L(h)$ in $\Omega_{1}^{\prime}:=\Omega \backslash \Omega_{0}$. In $\Omega_{1}^{\prime}, L(h)$ is expressed by using the coordinate $(l, \mu)$ of the tubular neighborhood as

$$
L(h) U=D \Delta_{l, \mu} U+F^{\prime}(S(r)) U+\delta K^{\delta}(l, \mu) U=A U+\delta K^{\delta} U
$$

for $(l, \mu) \in I_{\delta}:=\left[0, s_{0} / \delta\right] \times\left[0, N_{1} / \sqrt{\delta}\right)$ with the Neumann boundary condition at $\mu=0$ and the periodic boundary condition with respect to $l$. Here we note that the estimate $\left\|\delta K^{\delta} U\right\|=O(\sqrt{\delta})\|U\|_{C^{2}\left(I_{\delta}\right)}$ holds. Hence we appropriately extend the operator $A U+\delta K^{\delta} U$ to the one in $\boldsymbol{R}_{+}^{2}$ by extending the operator $\delta K^{\delta} U$ to $\boldsymbol{R}_{+}^{2}$ satisfying this estimate, which can be done by multiplying $K^{\delta}$ by cut-off functions disappearing outside of $I_{\delta}$.

Let $A_{1}(h):=A+\delta K^{\delta}$ in $C_{\text {unif }}\left(\boldsymbol{R}_{+}^{2}\right)$. Since $A$ is sectorial as an operator in $C_{\text {unif }}\left(\boldsymbol{R}_{+}^{2}\right),\left\|(\lambda-A)^{-1}\right\| \leq c_{3} /|\lambda|$ for $\lambda \in \rho(A)$, where $\rho(A)$ denotes the resolvent set of $A$. Now we need the following proposition.

\section{Proposition 4.1}

$$
\left\|\delta(\lambda-A)^{-1} K^{\delta}\right\| \leq \sqrt{\delta} c_{3}\left(1+\frac{1}{|\lambda|}\right)
$$

for $\lambda \in \rho(A)$ with $|\lambda| \geq c_{4} \sqrt[4]{\delta}$.

Proof Let $(\lambda-A)^{-1}\left(\delta K^{\delta}\right) g=U$. Then $\delta K^{\delta} g=(\lambda-A) U . \delta K^{\delta} g$ contains terms of $g_{z}, g_{l l}$, and so on, with $O(\sqrt{\delta})$ coefficients. For example, let us consider $a(l, \mu) g_{l l}=$ $(\lambda-A) U$ for some function $a=a(l, \mu) \in \boldsymbol{R}$ with $a=O(\sqrt{\delta})$. Set $U^{*}:=$ $(\lambda-A)^{-1}(a g)$. Then $(\lambda-A) U_{l l}^{*}=a g_{l l}+2 a_{l} g_{l}+a_{l l} g+\left(F^{\prime}(S(r)) U^{*}\right)_{l l}-F^{\prime}(S(r)) U_{l l}^{*}$. 
Similarly, setting $U^{* *}:=(\lambda-A)^{-1}\left(a_{l} g\right)$, we have $(\lambda-A) U_{l}^{* *}=a_{l} g_{l}+a_{l l} g+$ $\left(F^{\prime}(S(r)) U^{* *}\right)_{l}-F^{\prime}(S(r)) U_{l}^{* *}$. Then $(\lambda-A)\left(U-U_{l l}^{*}+2 U_{l}^{* *}\right)=g^{*}$ and $U$ is given by $U=U_{l l}^{*}-2 U_{l}^{* *}+(\lambda-A)^{-1} g^{*}$, where $g^{*}:=a_{l l} g-\left(F^{\prime}(S(r)) U^{*}\right)_{l l}+$ $F^{\prime}(S(r)) U_{l l}^{*}+2\left(F^{\prime}(S(r)) U^{* *}\right)_{l}-2 F^{\prime}(S(r)) U_{l}^{* *}$. Since $U_{l l}^{*}, U_{l}^{* *}$, and $g^{*}$ are estimated by $\sqrt{\delta} c_{4}\left(1+\frac{1}{|\lambda|}\right)\|g\|,\|U\| \leq \sqrt{\delta} c_{5}\left(1+\frac{1}{|\lambda|}\right)\|g\|$. All other cases are treated similarly.

Let us come back to the proof of Lemma 4.1. We consider $\left(\lambda-A_{1}(h)\right) U=g$ for $\lambda \in \rho(A)$. Since $\lambda \in \rho(A)$,

$$
\left\{I d-\delta(\lambda-A)^{-1} K^{\delta}\right\} U=(\lambda-A)^{-1} g .
$$

If the inequality $|\lambda| \geq c_{6} \sqrt[4]{\delta}$ holds, Proposition 4.1 implies

$$
\left\|\delta(\lambda-A)^{-1} K^{\delta}\right\| \leq \sqrt{\delta} c_{3}\left(1+\frac{1}{|\lambda|}\right) \leq c_{7}(\sqrt{\delta}+\sqrt[4]{\delta})
$$

because $\delta K^{\delta}=O(\sqrt{\delta})$. Thus $\left\{I d-\delta(\lambda-A)^{-1} K^{\delta}\right\}$ is invertible, and $U$ is given by

$$
U=\left\{I d-\delta(\lambda-A)^{-1} K^{\delta}\right\}^{-1}(\lambda-A)^{-1} g,
$$

which shows

$$
\left\|\left(\lambda-A_{1}(h)\right)^{-1}\right\| \leq \frac{c_{8}}{|\lambda|}
$$

for $\lambda \in \rho(A)$ with $|\lambda| \geq c_{6} \sqrt[4]{\delta}$. Here we note that $A_{1}(h)$ is an operator in $C_{\text {unif }}\left(\boldsymbol{R}_{+}^{2}\right)$. Since $s_{0} / \delta$ is sufficiently large, we may assume (4.1) holds for $A_{1}(h)$ in $\boldsymbol{R}_{+}^{\prime}:=\{0 \leq$ $\left.\mu<\infty, 0 \leq l \leq s_{0} / \delta\right\}$.

Let $L_{0}:=\delta^{2} D \Delta+F^{\prime}(\mathbf{0})$ in $\Omega$, and $L_{1}(h)$ of $(x, y) \in \Omega_{1}$ be the operator $A_{1}(h)$ of $(l, \mu) \in C_{\text {unif }}\left(\boldsymbol{R}_{+}^{\prime}\right)$, expressed with the original coordinates $s=\delta l+h$ and $z=\delta \mu$. We may assume $L_{1}(h)$ is defined in $\Omega_{3}:=\Omega\left(3 N_{1} \sqrt{\delta}\right)$. Define $D(\lambda):=$ $\chi_{1}(\boldsymbol{x})\left(\lambda-L_{1}(h)\right)^{-1} \tilde{\chi}_{1}(\boldsymbol{x})+\chi_{0}(\boldsymbol{x})\left(\lambda-L_{0}\right)^{-1}$, where $\tilde{\chi}_{1}(\boldsymbol{x})$ is a cut-off function satisfying $0 \leq \widetilde{\chi}_{1}(\boldsymbol{x}) \leq 1, \widetilde{\chi}_{1}(\boldsymbol{x})=1$ for $\boldsymbol{x} \in \Omega_{1}$, and $\widetilde{\chi}_{1}(\boldsymbol{x})=0$ for $\boldsymbol{x} \in \Omega \backslash \Omega_{3}$. Here we note that $L(h)=L_{1}(h)$ in $\Omega_{1}^{\prime}$ and

$$
(\lambda-L(h)) D(\lambda)=I d
$$

in $\Omega_{1}^{\prime} \cup \Omega_{0}^{\prime}$, where $\Omega_{0}^{\prime}:=\Omega \backslash \Omega_{1}$. Since $\Omega=\Omega_{1}^{\prime} \cup \Omega_{0}^{\prime} \cup \Omega_{2}$, it suffices to consider in $\Omega_{2}$. Let $U_{0}:=\left(\lambda-L_{0}\right)^{-1} g$ and $U_{1}:=\left(\lambda-L_{1}(h)\right)^{-1} \tilde{\chi}_{1} g$.

Proposition 4.2 If $\operatorname{Re} \lambda>-\gamma_{1}$ for $\gamma_{1}>0$, then

$$
\left\|U_{1}-U_{0}\right\|_{C^{2}\left(\Omega_{2}\right)} \leq c_{10} e^{-c_{9} / \sqrt{\delta}}\left(\left\|U_{0}\right\|_{C^{0}\left(\Omega_{3}\right)}+\left\|U_{1}\right\|_{C^{0}\left(\Omega_{3}\right)}\right)
$$

for $c_{9}, c_{10}>0$.

The proof of this proposition will be shown in the appendix. 
We can write $U_{0}=U_{1}+B_{0}(h) U_{0}+B_{1}(h) U_{1}$ with $\left\|B_{j}(h)\right\| \leq O\left(e^{-c_{9} / \sqrt{\delta}}\right)$ in $\Omega_{2}$. Hence it follows in $\Omega_{2}$

$$
(\lambda-L(h)) D(\lambda) g=(\lambda-L(h))\left(\chi_{1} U_{1}+\chi_{0} U_{0}\right)=g+B_{2}(h) g,
$$

where $B_{2}(h) g:=(\lambda-L(h)) \chi_{0}\left\{B_{0}(h)\left(\lambda-L_{0}\right)^{-1} g+B_{1}(h)\left(\lambda-L_{1}(h)\right)^{-1} \tilde{\chi}_{1} g\right\}$ with $\left\|B_{2}(h)\right\| \leq O\left(e^{-c_{9} / \sqrt{\delta}}\right)$. We may assume $B_{2}(h)$ is defined in $\Omega$ with the same estimate. Then from (4.2), it follows in $\Omega$

$$
(\lambda-L(h)) D(\lambda) g=g+B_{2}(h) g .
$$

Since $\left\|B_{2}(h)\right\| \leq O\left(\frac{e^{-c_{9} / \sqrt{\delta}}}{|\lambda|}\right) \leq O\left(\frac{e^{-c_{9} / \sqrt{\delta}}}{\sqrt[4]{\delta}}\right) \ll 1$ for $|\lambda| \geq c_{11} \sqrt[4]{\delta},\left(I d+B_{2}(h)\right)$ is invertible. Thus we find that

$$
(\lambda-L(h)) D(\lambda)\left(I d+B_{2}(h)\right)^{-1}=I d
$$

and

$$
(\lambda-L(h))^{-1}=D(\lambda)\left(I d+B_{2}(h)\right)^{-1}=D(\lambda)\left(I d+B_{3}(h)\right),
$$

where $B_{3}(h):=\left(I d+B_{2}(h)\right)^{-1}-I d$ with $\left\|B_{3}(h)\right\| \leq O\left(e^{-c_{9} / \sqrt{\delta}}\right)$. This shows $\left\{|\lambda| \geq c_{11} \sqrt[4]{\delta}\right\} \cap\left\{\operatorname{Re} \lambda>-\gamma_{1}\right\} \subset \rho(L(h))$.

Let $X^{\omega}$ be the fractional powered space of $X$ for $1 / 2<\omega<1$. Then we note that $\|\nabla U\| \leq c\|U\|_{\omega}$ for $c>0$, where $\|\cdot\|_{\omega}$ is the norm of $X^{\omega}$. Let $Q(h):=$ $\frac{1}{2 \pi} \int_{C_{0}}(\lambda-L(h))^{-1} d \lambda$ and $R(h):=I d-Q(h)$, where $C_{0}$ is a circle surrounding $I_{0}(h)$ in the region $\left\{\operatorname{Re} \lambda>-\gamma_{1}\right\}$. Then in a way quite similar to Lemma 5.2 in [6], $Q(h) U=\frac{2}{\pi \delta^{2}}\left\langle U,\left.\boldsymbol{\phi}^{*}(h)\right|_{2} \boldsymbol{\phi}(\boldsymbol{x} ; h)+B_{4}(h) U\right.$ with $\left\|B_{4}(h)\right\| \leq O(\delta)$, and $I_{0}(h)=\left\{\lambda_{0}(h)\right\}$ with $\left|\lambda_{0}(h)\right| \leq O(\delta)$, where $\boldsymbol{\phi}(h)(\boldsymbol{x}):=\chi_{1}(\boldsymbol{x}) \partial_{l} S(r(\boldsymbol{x} ; h) / \delta)$ and $\boldsymbol{\phi}^{*}(h)(\boldsymbol{x}):=\chi_{1}(\boldsymbol{x}) \boldsymbol{\phi}^{*}(r(\boldsymbol{x} ; h) / \delta)$. Let $\varphi(h)$ and $\varphi^{*}(h)$ be eigenfunctions corresponding to the spectral set $I_{0}(h)$ of $L(h)$ and the adjoint operator $L^{*}(h)$, respectively satisfying $\varphi(h)(\boldsymbol{x})=\boldsymbol{\phi}(h)(\boldsymbol{x})+O(\delta)$ and $\varphi^{*}(h)(\boldsymbol{x})=\boldsymbol{\phi}^{*}(h)(\boldsymbol{x})+O(\delta)$. Note that $\left\langle\boldsymbol{\phi}(h), \varphi^{*}(h)\right\rangle_{2}=\frac{\pi}{2} \delta^{2}+O\left(\delta^{3}\right)$. Set $E(h):=Q(h) X$ and $E^{\perp}(h):=R(h) X$. Now we can construct a map $\Pi(h): E^{\perp}\left(h_{0}\right) \rightarrow E^{\perp}(h)$ such that

$$
\|\Pi(h)\|,\left\|\Pi^{-1}(h)\right\| \leq c,\left\|\Pi_{h}(h) W\right\| \leq c\|\nabla W\|
$$

for $0 \leq h \leq s_{0}$ (periodic with respect to $h$ ). This is proved in Appendices.

Define $\boldsymbol{\Xi}(\boldsymbol{x} ; h):=\chi_{1}(\boldsymbol{x}) V_{1}(l, \mu)$ for $l=(\Sigma(\boldsymbol{x})-h) / \delta$ and $\mu=Z(\boldsymbol{x}) / \delta$, where $V_{1}(l, \mu) \in E^{\perp}$ is the function defined in (3.4).

Defining $S(\boldsymbol{x} ; h, \delta):=S(\boldsymbol{x} ; h)+\delta \boldsymbol{\Xi}(\boldsymbol{x} ; h)$ and substituting $U=S(\boldsymbol{x} ; h, \delta)+$ $\Pi(h) W$ for $W \in E^{\perp}\left(h_{0}\right)$, we have

$$
h_{t}\left(S_{h}(h, \delta)+\Pi_{h}(h) W\right)+\Pi(h) W_{t}=\mathcal{L}(S(h, \delta))+L(h) \Pi(h) W+G(W, h),
$$

with $|G(W, h)| \leq c|W|^{2}$. Define $W\left(D_{1}\right):=\left\{W \in E^{\perp}\left(h_{0}\right) \cap X^{\omega} ;\|W\|_{\omega} \leq\right.$ $\left.D_{1} \delta^{2}\right\}$ and $W\left(D_{1}, D_{2}\right):=\left\{W \in C\left(\left[0, s_{0}\right] ; \quad E^{\perp}\left(h_{0}\right)\right) ;\|W(h)\|_{\omega} \leq D_{1} \delta^{2}\right.$, 
$\left.\|W(h)-W(k)\|_{\omega} \leq D_{2} \delta|h-k|\right\}$. Since $\partial_{h} S(\boldsymbol{x} ; h)=-\frac{s-h}{\delta r(\boldsymbol{x} ; h)} S_{r}(r(\boldsymbol{x} ; h) / \delta)+O(\delta)=$ $-\frac{1}{\delta} \partial_{l} S(r(\boldsymbol{x} ; h) / \delta)+O(\delta)$, we have $\left\langle\partial_{h} S(h, \delta), \varphi^{*}(h)\right\rangle_{2}=-\frac{\pi}{2} \delta+O\left(\delta^{2}\right)$. Then for $W \in W\left(D_{1}\right)$ operating $Q(h)$ on (4.5), we have

$$
h_{t}\left(-\frac{\pi}{2} \delta+O\left(\delta^{2}\right)\right)=\left\langle\mathcal{L}(S(h, \delta)), \boldsymbol{\phi}^{*}(h)\right\rangle_{2}+O\left(\delta^{6}\right)
$$

because $\varphi^{*}(h)(\boldsymbol{x})$ is a function of the form $E(r(\boldsymbol{x} ; h) / \delta, \theta)$ for some function $E(r, \theta)$ decaying exponentially with respect to $r$. Hence we can write $h_{t}=G_{1}(W, h)$ satisfying $G_{1}(W, h)=-\frac{2}{\pi \delta}\left\langle\mathcal{L}(S(h, \delta)), \phi^{*}(h)\right\rangle_{2}+O\left(\delta^{5}\right)$. Since

$$
\left\langle\mathcal{L}(S(h, \delta)), \phi^{*}(h)\right\rangle_{2}=-\delta^{4} \frac{4}{15} M_{0} \kappa_{s}(h)+O\left(\delta^{5}\right)
$$

by the calculation in Sect. 3, we have

$$
G_{1}(W, h)=\delta^{3} \frac{8}{15 \pi} M_{0} \kappa_{S}(h)+O\left(\delta^{4}\right)
$$

for $W \in W\left(D_{1}\right)$.

On the other hand, operating $R(h)$ on (4.5), we have

$$
\begin{aligned}
h_{t} O\left(\delta^{2}\right)+\Pi(h) W & =R(h) \mathcal{L}(S(h, \delta))+L(h) \Pi(h) W+R(h) G(W, h) \\
& =L(h) \Pi(h) W+O\left(\delta^{2}\right) .
\end{aligned}
$$

Thus, (4.5) is written as

$$
\left\{\begin{array}{l}
h_{t}=G_{1}(h, W), \\
W_{t}=\widehat{L}(h) W+G_{2}(h, W),
\end{array}\right.
$$

where $\widehat{L}(h):=\Pi^{-1}(h) L(h) \Pi(h)$ and $G_{2}(h, W):=\Pi^{-1}(h)\{R(h) \mathcal{L}(S(h, \delta))+$ $\left.R(h) G(W, h)-G_{1}(h, W) R(h) S_{h}\right\}=O\left(\delta^{2}\right)$. Moreover it follows that for $W$, $V \in W\left(D_{1}\right)$,

$$
\begin{aligned}
\|\widehat{L}(h)-\widehat{L}(k)\| & \leq \frac{c_{12}}{\delta}|h-k|, \\
\left|G_{1}(h, W)-G_{1}(k, V)\right| & \leq c_{13} \delta^{2}\left\{|h-k|+\|W-V\|_{\omega}\right\}, \\
\left\|G_{2}(h, W)-G_{2}(k, V)\right\| & \leq c_{14} \delta\left\{|h-k|+\|W-V\|_{\omega}\right\} .
\end{aligned}
$$

Combining the above estimates and (4.4), we can show the existence of an attractive invariant manifold $\mathcal{M}:=\left\{W=\sigma^{*}(h) \in W\left(D_{1}, D_{2}\right) ; 0 \leq h \leq s_{0}\right\}$ in (4.7) for an appropriately taken $D_{1}, D_{2}$ in a way quite similar to [6]. That is, the solution $U(t, \boldsymbol{x})$ of (1.2) is given by

$$
U(t, \boldsymbol{x})=S(\boldsymbol{x} ; h(t))+\delta \Xi(\boldsymbol{x} ; h(t))+\Pi(h(t)) \sigma^{*}(h(t))(\boldsymbol{x}),
$$

which completes the proof of the theorem. 


\subsection{Proof of Corollary 2.1}

In the first equation of (4.7), $h$ satisfies

$$
h_{t}=G_{1}\left(h, \sigma^{*}(h)\right)=\delta^{3} G_{1}^{*}\left(h, \sigma^{*}(h)\right)=: \delta^{3} G^{* *}(h ; \delta) .
$$

Since $\sigma^{*} \in W\left(D_{1}, D_{2}\right)$, a stable (or unstable ) equilibrium $h^{*}$ of $\frac{8}{15 \pi} M_{0} \kappa_{S}(h)=$ $G_{1}^{* *}(h ; 0)$, in the linearized sense, generates a stable (or unstable ) equilibrium of $G^{* *}(h ; \delta)$ by the implicit function theorem for small $\delta>0$, which completes the proof.

\section{Proofs of Theorem 2.2 and Corollary 2.2}

\subsection{Proof of Theorem 2.2}

If $h:=h_{2}-h_{1}>\beta \delta|\log \delta|$ for a large $\beta>0$, then we have $\sqrt{\frac{\delta}{h}} e^{-\alpha h / \delta} \leq O\left(\delta^{4}\right)$, which trivially implies the equation (2.2). Hence it suffices to consider the case when $\beta_{1} \delta|\log \delta| \leq h=h_{2}-h_{1} \leq \beta_{2} \delta|\log \delta|$ for $0<\beta_{1}<\beta_{2}$.

Let $S\left(\boldsymbol{x} ; h_{1}, h_{2}\right):=S\left(\boldsymbol{x} ; h_{1}\right)+S\left(\boldsymbol{x} ; h_{2}\right)$ and $L\left(h_{1}, h_{2}\right):=\mathcal{L}_{\delta}^{\prime}\left(S\left(\boldsymbol{x} ; h_{1}, h_{2}\right)\right)=$ $\delta^{2} \Delta+F^{\prime}\left(S\left(\boldsymbol{x} ; h_{1}, h_{2}\right)\right)$. Taking the tubular coordinate $(l, \mu)$ defined by $s=\Sigma(\boldsymbol{x}), z=$ $Z(\boldsymbol{x})$ and $\delta l:=s-h_{1}(t), \delta \mu:=z$ and letting $S(l, \mu):=S(r)$ with $r:=\sqrt{l^{2}+\mu^{2}}$, we have $S\left(\boldsymbol{x} ; h_{1}, h_{2}\right)=S(l, \mu)+S(l-h / \delta, \mu)=: S(l, \mu ; h)$ and

$$
\begin{aligned}
L\left(h_{1}, h_{2}\right) U= & D\left\{U_{\mu \mu}-\frac{\delta \kappa}{1-\delta \mu \kappa} U_{\mu}+\frac{1}{1-\delta \mu \kappa}\left(\frac{1}{1-\delta \mu \kappa} U_{l}\right)_{l}\right\} \\
& +F^{\prime}(S(l, \mu ; h)) U \\
= & D \Delta_{l, \mu} U+F^{\prime}(S(l, \mu ; h)) U+\delta K^{\delta}(l, \mu) U
\end{aligned}
$$

in $\Omega_{1}$, where $\kappa=\kappa\left(h_{1}+\delta l\right)$. The inequality $\beta_{1} \delta|\log \delta| \leq h=h_{2}-h_{1} \leq \beta_{2} \delta|\log \delta|$ leads to $\beta_{1}|\log \delta| \leq h / \delta \leq \beta_{2}|\log \delta|$.

On the other hand, $L\left(h_{1}, h_{2}\right)=L_{0}+O\left(e^{-c / \sqrt{\delta}}\right)$ in $\Omega_{0}$. Then in quite a similar manner to the proof of Lemma 4.1, we can show

Lemma 5.1 The spectral set, say $I\left(h_{1}, h_{2}\right)$ of $L\left(h_{1}, h_{2}\right)$, is given by $I\left(h_{1}, h_{2}\right)=$ $I_{0}\left(h_{1}, h_{2}\right) \cup I_{1}\left(h_{1}, h_{2}\right)$, where $I_{0}\left(h_{1}, h_{2}\right) \subset\{|\lambda| \leq c \sqrt[4]{\delta}\}$ and $I_{1}\left(h_{1}, h_{2}\right) \subset\{$ Re $\lambda<$ $\left.-\gamma_{1}\right\}$ for positive constants $c$ and $\gamma_{1}$.

The rest of the proof is completed by combining the proofs of Theorem $2.1 \quad[6,8]$. In fact, we can show the existence of an exponentially attractive local invariant manifold $\mathcal{M}:=\left\{S\left(\boldsymbol{x} ; h_{1}, h_{2}\right)+\sigma^{*}\left(h_{1}, h_{2}\right)(\boldsymbol{x}) ; c_{1}<h_{1}<h_{2}<s_{0}-c_{1}, c_{2}|\delta \log \delta|<\right.$ $\left.h_{2}-h_{1}<\frac{1}{2} c_{1}\right\}$ with $\left\|\sigma^{*}\left(h_{1}, h_{2}\right)\right\|_{\omega} \leq O(\delta)$.

\subsection{Proof of Corollary 2.2}

We omit the details here, but the proof is quite similar to the proof of Corollary 2.1. We substitute $S\left(\boldsymbol{x} ; h_{1}, h_{2}\right)+\sigma^{*}\left(h_{1}, h_{2}\right)(\boldsymbol{x})$ into the equations $\partial_{t} h_{1}$ and $\partial_{t} h_{2}$ and use the implicit function theorem. 


\subsection{Proof of Corollary 2.3}

The positivity of the constant $M_{1}$ was proved in [8]. Now we calculate the value of the constant $M_{0}$.

Under the assumptions of the corollary, there exists a spike solution $S$ of (1.5) of the form $S(r)=(U(r / \varepsilon), V(r))$ in $\boldsymbol{R}_{+}^{2}$, where $r:=\sqrt{l^{2}+\mu^{2}}$, and functions $U(\zeta)$ and $V(r)$ are exponentially decaying positive functions. The function $\Phi^{*}(r)$ in the definition of the constant $M_{0}$ is given by

$$
\Phi^{*}(r)=\frac{1}{\int_{0}^{\infty} \rho\left(U_{\rho}\right)^{2} d \rho}(U(r / \varepsilon), 0)+o(1)
$$

as $\varepsilon \downarrow 0$ by the result of [8]. Hence the approximate value of $M_{0}$ is calculated as

$$
M_{0}=\frac{8}{15 \pi} \varepsilon\left(\frac{5 \int_{0}^{\infty} \rho^{2}\left(U_{\rho}\right)^{2} d \rho}{2 \int_{0}^{\infty} \rho\left(U_{\rho}\right)^{2} d \rho}+o(1)\right)>0
$$

Assumptions (H1)-(H3) are also discussed in [8], but on the whole $\boldsymbol{R}^{2}$ space. Then (H1)-(H3) in this paper are easily checked by the restriction to $\boldsymbol{R}_{+}^{2}$. In fact, the semisimpleness of the 0 eigenvalue in $\boldsymbol{R}^{2}$ [8] directly leads to (H2) and (H3).

\section{Analysis of ODE (2.3)}

In this section, we give the proof of the following theorem.

Theorem 6.1 Equation (2.3) has a linearly stable unique equilibrium, say $P=\left(p_{1}, p_{2}\right)$, if both $M_{0}$ and $M_{1}$ are positive.

Proof With the loss of generality, we may assume $\Gamma(0) \in \partial \Omega$ is a point of maximal curvature. Equilibria of (2.3) satisfy

$$
\left\{\begin{array}{l}
0=M_{0} \delta^{3} \frac{d \kappa}{d s}\left(p_{1}\right)-M_{1} \sqrt{\frac{\delta}{p}} e^{-\alpha p / \delta} \\
0=M_{0} \delta^{3} \frac{d \kappa}{d s}\left(p_{2}\right)+M_{1} \sqrt{\frac{\delta}{p}} e^{-\alpha p / \delta}
\end{array}\right.
$$

where $p=p_{2}-p_{1}$. Equation (6.1) leads to

$$
\frac{d \kappa}{d s}\left(p_{1}\right)+\frac{d \kappa}{d s}\left(p_{2}\right)=0
$$


Since $p_{1}, p_{2}$ are small, we can expand $\kappa_{s}\left(p_{1}\right)$ and $\kappa_{s}\left(p_{2}\right)$ as

$$
\left\{\begin{array}{l}
\frac{d \kappa}{d s}\left(p_{1}\right)=\frac{d \kappa}{d s}(0)+p_{1} \frac{d^{2} \kappa}{d s^{2}}(0)+\frac{1}{2} p_{1}^{2} \frac{d^{3} \kappa}{d s^{3}}(0)+O\left(p_{1}^{3}\right) \\
\frac{d \kappa}{d s}\left(p_{2}\right)=\frac{d \kappa}{d s}(0)+p_{2} \frac{d^{2} \kappa}{d s^{2}}(0)+\frac{1}{2} p_{2}^{2} \frac{d^{3} \kappa}{d s^{3}}(0)+O\left(p_{2}^{3}\right)
\end{array}\right.
$$

Since $p$ is also small, $p_{1}$ and $p_{2}$ can be expanded respectively as $p_{1}=\left(a_{1}-1\right) p+$ $a_{2} p^{2}+O\left(p^{3}\right), p_{2}=a_{1} p+a_{2} p^{2}+O\left(p^{3}\right)$, for some constants $a_{1}$ and $a_{2}$. Therefore (6.3) is rewritten as

$$
\left\{\begin{array}{l}
\frac{d \kappa}{d s}\left(p_{1}\right)=\kappa_{0}^{(2)}\left\{\left(a_{1}-1\right) p+a_{2} p^{2}\right\}+\frac{1}{2} \kappa_{0}^{(3)}\left(a_{1}-1\right)^{2} p^{2}+O\left(p^{3}\right) \\
\frac{d \kappa}{d s}\left(p_{2}\right)=\kappa_{0}^{(2)}\left\{a_{1} p+a_{2} p^{2}\right\}+\frac{1}{2} \kappa_{0}^{(3)} a_{1}^{2} p^{2}+O\left(p^{3}\right)
\end{array}\right.
$$

where

$$
\frac{d \kappa}{d s}(0)=0, \quad \kappa_{0}^{(2)}:=\frac{d^{2} \kappa}{d s^{2}}(0)<0, \quad \kappa_{0}^{(3)}:=\frac{d^{3} \kappa}{d s^{3}}(0)
$$

Thus (6.2) is rewritten as

$$
\left(2 \kappa_{0}^{(2)} a_{1}-\kappa_{0}^{(2)}\right) p+\left(2 \kappa_{0}^{(2)} a_{2}+\kappa_{0}^{(3)} a_{1}^{2}-\kappa_{0}^{(3)} a_{1}+\frac{1}{2} \kappa_{0}^{(3)}\right) p^{2}+O\left(p^{3}\right)=0
$$

Then $a_{1}$ and $a_{2}$ are determined by

$$
2 \kappa_{0}^{(2)}\left(a_{1}-\frac{1}{2}\right)=0, \quad 2 \kappa_{0}^{(2)} a_{2}+\kappa_{0}^{(3)}\left(a_{1}^{2}-a_{1}+\frac{1}{2}\right)=0
$$

and hence

$$
a_{1}=\frac{1}{2}, \quad a_{2}=-\frac{1}{8} \frac{\kappa_{0}^{(3)}}{\kappa_{0}^{(2)}}
$$

and

$$
p_{2}=\frac{1}{2} p-\frac{1}{8} \frac{\kappa_{0}^{(3)}}{\kappa_{0}^{(2)}} p^{2}+O\left(p^{3}\right)
$$

Here,

$$
M_{0} \delta^{3}\left(\kappa_{0}^{(2)} p_{2}+\frac{1}{2} \kappa_{0}^{(3)} p_{2}^{2}\right)+M_{1} \sqrt{\frac{\delta}{p}} e^{-\alpha p / \delta}+O\left(\delta^{3} p_{2}^{3}\right)=0
$$


holds by (6.1) and (6.3). Substituting (6.4) into (6.5), it follows that

$M_{0} \delta^{3}\left\{\kappa_{0}^{(2)}\left(\frac{1}{2} p-\frac{1}{8} \frac{\kappa_{0}^{(3)}}{\kappa_{0}^{(2)}} p^{2}\right)+\frac{1}{2} \kappa_{0}^{(3)}\left(\frac{1}{2} p\right)^{2}\right\}+M_{1} \sqrt{\frac{\delta}{p}} e^{-\alpha p / \delta}+O\left(\delta^{3} p^{3}\right)=0$

and hence

$$
\frac{1}{2} M_{0} \delta^{3} \kappa_{0}^{(2)} p+M_{1} \sqrt{\frac{\delta}{p}} e^{-\alpha p / \delta}+O\left(\delta^{3} p^{3}\right)=0 .
$$

Specially, the lowest-order part of (6.6) is

$$
\frac{1}{2} M_{0} \delta^{3} \kappa_{0}^{(2)} p+M_{1} \sqrt{\frac{\delta}{p}} e^{-\alpha p / \delta}=0
$$

Proposition 6.1 Equation (6.7) has a unique solution.

Proof Equation (6.7) can be rewritten as

$$
\frac{1}{2} M_{0} \delta^{4} \kappa_{0}^{(2)} \frac{p}{\delta}=-M_{1} \sqrt{\frac{1}{\frac{p}{\delta}}} e^{-\alpha p / \delta}
$$

Define $f(x):=\frac{1}{2} M_{0} \delta^{4} \kappa_{0}^{(2)} x, g(x):=-M_{1} x^{-1 / 2} e^{-\alpha x}$ for $x>0$. Then $\kappa_{0}^{(2)}<0$ yields a monotone decrease of $f(x)$. On the other hand, $g^{\prime}(x)=M_{1} x^{-3 / 2} e^{-\alpha x}\left(\frac{1}{2}+\right.$ $\alpha x)>0$ leads to the monotone increase of $g(x)$. Since $\lim _{x \rightarrow 0} f(x)=$ $0, \lim _{x \rightarrow \infty} f(x)=-\infty, \lim _{x \rightarrow 0} g(x)=-\infty$, and $\lim _{x \rightarrow \infty} g(x)=0$, we use the intermediate value theorem to show that there is only one intersection of $f(x)$ and $g(x)$. Thus (6.7) has a unique solution.

Proposition $6.2 p=O(\delta \log \delta)$.

Proof Putting $\tilde{p}=p / \delta$, we have

$$
-\frac{1}{2} M_{0} \delta^{4} \kappa_{0}^{(2)} \tilde{p}=M_{1} \sqrt{\frac{1}{\tilde{p}}} e^{-\alpha \tilde{p}}(>0)
$$

by (6.7). Suppose $\lim _{\delta \rightarrow 0} \tilde{p}=0$. Then the right hand side of (6.8) goes to $\infty$ as $\delta \rightarrow 0$. But this means that $\lim _{\delta \rightarrow 0} \delta^{4} \tilde{p}=\infty$, which leads to the contraction. Therefore $\lim _{\delta \rightarrow 0} \tilde{p} \neq 0$. That is, there is a positive constant $C>0$ such that $\tilde{p} \geq C$ and

$$
-\frac{1}{2} M_{0} \delta^{4} \kappa_{0}^{(2)} C \leq-\frac{1}{2} M_{0} \delta^{4} \kappa_{0}^{(2)} \tilde{p}=M_{1} \sqrt{\frac{1}{\tilde{p}}} e^{-\alpha \tilde{p}} \leq M_{1} \sqrt{\frac{1}{C}} e^{-\alpha \tilde{p}}
$$


Setting $C_{1}:=-\frac{1}{2} \frac{M_{0}}{M_{1}} \kappa_{0}^{(2)} C \sqrt{C}(>0)$, we have $C_{1} \delta^{4} \leq e^{-\alpha \tilde{p}}$ and

$$
\tilde{p} \leq-\frac{1}{\alpha} \log C_{1}+\frac{4}{\alpha}|\log \delta| \leq C_{2}|\log \delta|
$$

for a constant $C_{2}>0$. Noting

$$
e^{-2 \alpha \tilde{p}} \leq \sqrt{\frac{1}{\tilde{p}}} e^{-\alpha \tilde{p}}
$$

by the inequality $e^{-\alpha x} \leq \sqrt{1 / x}(x>0)$, we have

$$
e^{-2 \alpha \tilde{p}} \leq \sqrt{\frac{1}{\tilde{p}}} e^{-\alpha \tilde{p}}=-\frac{1}{2} \frac{M_{0}}{M_{1}} \kappa_{0}^{(2)} \tilde{p} \delta^{4} \leq-\frac{1}{2} \frac{M_{0}}{M_{1}} \kappa_{0}^{(2)} C_{2} \delta^{3}(\delta|\log \delta|) .
$$

Setting $C_{3}:=-\frac{1}{2} \frac{M_{0}}{M_{1}} \kappa_{0}^{(2)} C_{2}(>0)$, we also have $e^{-2 \alpha \tilde{p}} \leq C_{3} \delta^{3}$ and

$$
\tilde{p} \geq-\frac{1}{2 \alpha} \log C_{3}+\frac{3}{2 \alpha}|\log \delta|
$$

Equations (6.9) and (6.10) complete the proof.

Proposition 6.3 $P=\left(p_{1}, p_{2}\right)$ is stable in the linearized sense.

Proof We define the right-hand side of (2.3) by $G\left(h_{1}, h_{2}\right), H\left(h_{1}, h_{2}\right)$ :

$$
\left\{\begin{array}{l}
H\left(h_{1}, h_{2}\right):=M_{0} \delta^{3} \frac{d \kappa}{d s}\left(h_{1}\right)-M_{1} \sqrt{\frac{\delta}{h}} e^{-\alpha h / \delta}, \\
G\left(h_{1}, h_{2}\right):=M_{0} \delta^{3} \frac{d \kappa}{d s}\left(h_{2}\right)+M_{1} \sqrt{\frac{\delta}{h}} e^{-\alpha h / \delta} .
\end{array}\right.
$$

Then, the linearized operator $L_{p}$ of (2.3) with respect to $P=\left(p_{1}, p_{2}\right)$ can be represented as

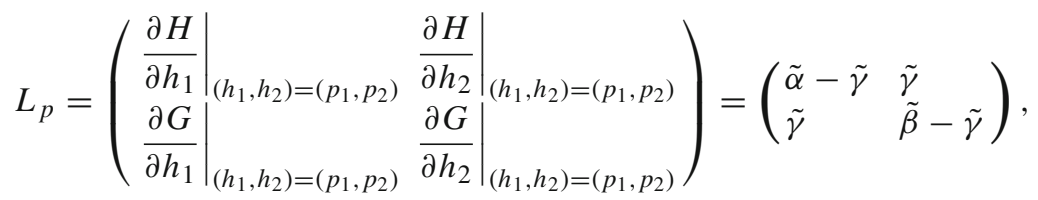

where

$$
\left\{\begin{array}{l}
\tilde{\alpha}:=M_{0} \delta^{3} \frac{d^{2} \kappa}{d s^{2}}\left(p_{1}\right)(<0) \\
\tilde{\beta}:=M_{0} \delta^{3} \frac{d^{2} \kappa}{d s^{2}}\left(p_{2}\right)(<0) \\
\tilde{\gamma}:=M_{1} \sqrt{\frac{\delta}{p}} e^{-\alpha p / \delta}\left(\frac{1}{2 p}+\frac{\alpha}{\delta}\right)(>0)
\end{array}\right.
$$


Thus, it follows $\operatorname{tr} L_{p}=(\tilde{\alpha}+\tilde{\beta})-2 \tilde{\gamma}<0$ and $\operatorname{det} L_{p}=\tilde{\alpha} \tilde{\beta}-(\tilde{\alpha}+\tilde{\beta}) \tilde{\gamma}>0$. Thus $P=\left(p_{1}, p_{2}\right)$ is stable in the linearized sense.

Acknowledgments This paper is supported by a Grant-in-Aid for Scientific Research (No. 21654019).

Open Access This article is distributed under the terms of the Creative Commons Attribution License which permits any use, distribution, and reproduction in any medium, provided the original author(s) and the source are credited.

\section{Appendices}

In this section, we give the proofs of Propositions 4.2 and (4.4).

\subsection{Proof of Proposition 4.2}

In this proof, we use the symbols $c, c^{\prime}, c^{\prime \prime}, \cdots$ as general positive constants independent of small $\delta>0$.

Let $\Omega_{1 / 2}:=\Omega\left(\frac{1}{2} N_{1} \sqrt{\delta}\right)$ and $\Omega_{0}^{\prime \prime}:=\Omega \backslash \Omega_{1 / 2}$. For any $\boldsymbol{x}_{0} \in \Omega_{2}$, we define two balls $B_{\delta r_{0}}\left(\boldsymbol{x}_{0}\right) \subset B_{\sqrt{\delta} r_{1}}\left(\boldsymbol{x}_{0}\right) \subset \Omega_{0}^{\prime \prime}$ for $r_{0}, r_{1}>0$, where $B_{r}\left(\boldsymbol{x}_{0}\right):=\left\{\boldsymbol{x} \in \Omega_{1} ;\left|\boldsymbol{x}-\boldsymbol{x}_{0}\right|<\right.$ $r$. Since $|S(\boldsymbol{x} ; h)| \leq O\left(e^{-c / \sqrt{\delta}}\right)$ in $\Omega_{0}^{\prime \prime}$ for $c>0, L_{1}(h)$ can be expressed as $L_{1}(h)=$ $L_{0}+C_{1}(h)$ with $\left|C_{1}(h)\right| \leq c e^{-c^{\prime} / \sqrt{\delta}}$ in $\Omega_{0}^{\prime \prime}$ for $c, c^{\prime}>0$. Then $\left(\lambda-L_{0}\right)\left(U_{1}-U_{0}\right)=$ $C_{1}(h) U_{1}$ in $B_{\sqrt{\delta} r_{1}}\left(x_{0}\right) \subset \Omega_{0}^{\prime \prime}$. Taking the stretched coordinate $\boldsymbol{\xi}:=\left(\boldsymbol{x}-\boldsymbol{x}_{0}\right) / \delta$, we see that the equation $\left(\lambda-L_{0}\right)\left(U_{1}-U_{0}\right)=C_{1}(h) U_{1}$ in $B_{\sqrt{\delta} r_{1}}\left(x_{0}\right)$ becomes

$$
\left(\lambda-A_{0}\right)\left(U_{1}-U_{0}\right)=C_{2}(h) U_{1}, \quad \xi \in B_{1}^{*},
$$

where $A_{0}:=D \Delta_{\xi}+F^{\prime}(\mathbf{0}), B_{1}^{*}:=B_{r_{1} / \sqrt{\delta}}(\mathbf{0})$ and $C_{2}(h)$ satisfies $\left|C_{2}(h)\right| \leq c e^{-c^{\prime} / \sqrt{\delta}}$ for $c, c^{\prime}>0$.

Since $\delta$ is sufficiently small, $B_{1}^{*}$ is nearly $\boldsymbol{R}^{2}$ and the assumption (H2)' says that $\left(\lambda-A_{0}\right)$ is also invertible in $X_{0}:=C_{\text {unif }}\left(\boldsymbol{R}^{2}\right)$ by taking $\lambda$ in the resolvent set of $A_{0}$. Here we note that the spectral set $I\left(A_{0}\right)$ is the same even if we consider that $A_{0}$ is in the whole space $\boldsymbol{R}^{2}$. Now we may extend $C_{2}(h), U_{0}$, and $U_{1}$ to $\boldsymbol{R}^{2}$ with similar estimates $\left|C_{2}(h)\right| \leq c e^{-c^{\prime} / \sqrt{\delta}},\left\|U_{0}\right\|_{X_{0}} \leq\left\|U_{0}\right\|_{C^{0}\left(B_{1}^{*}\right)} \leq\left\|U_{0}\right\|_{C^{0}\left(\Omega_{3}\right)}$, and $\left\|U_{1}\right\|_{X_{0}} \leq\left\|U_{1}\right\|_{C^{0}\left(B_{1}^{*}\right)} \leq\left\|U_{1}\right\|_{C^{0}\left(\Omega_{3}\right)}$, respectively, for $c, c^{\prime}>0$. Since $\left(\lambda-A_{0}\right)$ is invertible in $X_{0}$, there exist unique $W \in X_{0}$ satisfying $\left(\lambda-A_{0}\right) W=C_{2}(h) U_{1}$ in $\boldsymbol{R}^{2}$. Subtracting this equation from (7.1) on both sides, we have

$$
\left(\lambda-A_{0}\right)\left(U_{1}-U_{0}-W\right)=0, \quad \xi \in B_{1}^{*}
$$

Since $\operatorname{Re} \lambda>-\gamma_{1}, \operatorname{dist}\left\{\lambda, I\left(A_{0}\right)\right\}>\gamma_{2}$ for $\gamma_{2}>0$. Hence we have $W=$ $\left(\lambda-A_{0}\right)^{-1} C_{2}(h) U_{1}$ and

$$
\|W\|_{C^{2}\left(\boldsymbol{R}^{2}\right)} \leq c e^{-c^{\prime} / \sqrt{\delta}}\left\|U_{1}\right\|_{X_{0}} \leq c e^{-c^{\prime} / \sqrt{\delta}}\left\|U_{1}\right\|_{C^{0}\left(\Omega_{3}\right)}
$$


for $c, c^{\prime}>0$, and it suffices to show that

$$
\left\|U_{1}-U_{0}-W\right\|_{C^{2}\left(B_{0}^{*}\right)} \leq O\left(e^{-c^{\prime} / \sqrt{\delta}}\right)\left\|U_{1}-U_{0}-W\right\|_{C^{0}\left(B_{1}^{*}\right)},
$$

where $B_{0}^{*}:=B_{r_{0}}(\mathbf{0})$.

Let $V:=U_{1}-U_{0}-W$, and consider the equation

$$
\left(\lambda-A_{0}\right) V=0, \quad \xi \in B_{1}^{*}
$$

for $\lambda \in C$ with $\operatorname{Re} \lambda>-\gamma_{1}$.

First consider (7.4) in the interval $I_{1}^{*}:=\left(-r_{1} / \sqrt{\delta}, r_{1} / \sqrt{\delta}\right) \subset \boldsymbol{R}^{1}$. Then (7.4) corresponds to

$$
\lambda V-D V^{\prime \prime}-F_{0} V=\mathbf{0},
$$

where $F_{0}:=F^{\prime}(\mathbf{0})$. If $V(\xi)=e^{\mu \xi} \boldsymbol{b}$, then

$$
\left(\mu^{2} D+F_{0}-\lambda\right) \boldsymbol{b}=\mathbf{0}
$$

that is,

$$
\operatorname{det}\left(\mu^{2} D+F_{0}-\lambda\right)=0
$$

Lemma 7.1 $\mu$ in (7.6) satisfies $|\operatorname{Re} \mu| \geq \gamma_{3}$ for $\gamma_{3}>0$ independent of $\operatorname{Re} \lambda>-\gamma_{1}$.

Proof Define $\Xi(\tau):=-\tau D+F_{0}$, and let $I(\tau)$ be the set of eigenvalues of $\Xi(\tau)$. Then by using a Fourier transform, we see that the spectral set $I\left(A_{0}\right)$ is given by $I\left(A_{0}\right)=\cup_{\tau \geq 0} I(\tau)$.

Putting $\mu^{2}=a+i b(a, b \in \boldsymbol{R}, i:=\sqrt{-1})$ and substituting it into the eigenvalue equation (7.5), we have

$$
\left\{I+i b\left(a D+F_{0}-\lambda\right)^{-1} D\right\} \boldsymbol{b}=\mathbf{0}
$$

if $\left(a D+F_{0}-\lambda\right)^{-1}$ exists. Let $E(\lambda):=\left\{I+i b\left(a D+F_{0}-\lambda\right)^{-1} D\right\}$.

First, we consider the case $a \leq 0$. Put $a=-\tau(\tau \geq 0)$. In this case,

$$
\frac{c}{|\lambda|} \leq\left|\left(-\tau D+F_{0}-\lambda\right)^{-1}\right| \leq c^{\prime}
$$

for $c, c^{\prime}>0$ because of $\operatorname{dist}\left\{\lambda, I\left(A_{0}\right)\right\}>\gamma_{2}$. Hence if $|b|$ is sufficiently small, $E(\lambda)$ is invertible and $\boldsymbol{b}$ must be zero vector in (7.7). This contradicts (7.6). Thus, $|b| \geq \gamma_{4}$ for $\gamma_{4}>0$.

On the other hand, it follows that $\operatorname{dist}(\lambda, I(\tau)) \rightarrow \infty$ as $\tau \rightarrow \infty$ uniformly for $\operatorname{Re} \lambda>-\gamma_{1}$ and hence

$$
\left(-\tau D+F_{0}-\lambda\right)^{-1} \rightarrow \mathbf{0}
$$


as $\tau \rightarrow \infty$ uniformly for $\operatorname{Re} \lambda>-\gamma_{1}$, which means $E(\lambda)$ is invertible. Thus, $\tau$ must be $\tau \leq \gamma_{5}$ for $\gamma_{5}>0$. Since $R e \mu$ is given by

$$
|R e \mu|^{2}=\frac{b^{2}}{2 \tau+\sqrt{\tau^{2}+b^{2}}},
$$

the estimates $|b| \geq \gamma_{4}$ and $0 \leq \tau \leq \gamma_{5}$ imply $|\operatorname{Re} \mu| \geq \gamma_{3}$ for $\gamma_{3}>0$.

Next we consider the case $a>0$. In this case, $|\operatorname{Re} \mu|$ is given by

$$
|\operatorname{Re} \mu|^{2}=\frac{a+\sqrt{a^{2}+b^{2}}}{2} \geq \frac{1}{2}(a+|b|) .
$$

Therefore, it suffices to show $(a+|b|)>\gamma_{6}$ for $\gamma_{6}>0$.

Since $\operatorname{dist}\left\{\lambda, I\left(A_{0}\right)\right\}>\gamma_{2}$ for $\operatorname{Re} \lambda>-\gamma_{1}$, we have $\operatorname{dist}\left\{\lambda, I^{\prime}\left(A_{0}\right)\right\}>\gamma_{2}^{\prime}$, where $I^{\prime}\left(A_{0}\right):=\cup_{\tau \geq-\gamma_{7}} I(\tau)$ for $\gamma_{2}^{\prime}, \gamma_{7}>0$. Hence, $\left(a D+F_{0}-\lambda\right)^{-1}$ is uniformly bounded for $0 \leq a \leq \gamma_{7}$ and $\operatorname{Re} \lambda>-\gamma_{1}$. If $|b|$ is arbitrarily small, (7.7) leads to the invertibility of $E(\lambda)$. That is, if $0 \leq a \leq \gamma_{7}$ and $|b| \leq \gamma_{8}$ for $\gamma_{8}>0$, then $E(\lambda)$ is invertible, which contradicts (7.6). Thus, either $|b|>\gamma_{8}$ or $a>\gamma_{7}$. Then we have $(a+|b|)>\gamma_{6}$ for $\gamma_{6}>0$.

Finally, we consider (7.4) in $B_{1}^{*}$. Let $V=V(r, \theta)=\sum_{n=-\infty}^{\infty} \boldsymbol{b}_{n}(r) e^{i n \theta}$ and $g(\theta):=V \mid \partial B_{1}^{*}$, where $(r, \theta)$ is the polar coordinate of $B_{1}^{*}$ and $\boldsymbol{b}_{n}(r) \in \boldsymbol{R}^{N}$. Then (7.4) becomes

$$
\left\{\begin{array}{l}
\left(\boldsymbol{b}_{n}^{\prime \prime}+\frac{1}{r} \boldsymbol{b}_{n}^{\prime}-\frac{n^{2}}{r^{2}} \boldsymbol{b}_{n}\right)+D^{-1}\left(F_{0}-\lambda\right) \boldsymbol{b}_{n}=\mathbf{0}, \\
\boldsymbol{b}_{n}^{\prime}(0)=\mathbf{0}, \boldsymbol{b}_{n}\left(r_{1} / \sqrt{\delta}\right)=g_{n},
\end{array}\right.
$$

where $g(\theta)=\sum_{n=-\infty}^{\infty} g_{n} e^{i n \theta}$.

Let $\left\{\boldsymbol{e}_{j}\right\}$ be the bases of $\boldsymbol{R}^{N}$ consisting of the eigenvectors of $D^{-1}\left(F_{0}-\lambda\right)$. That is, $D^{-1}\left(K_{0}-\lambda\right) \boldsymbol{e}_{j}=-\mu_{j}^{2} \boldsymbol{e}_{j}$ are satisfied. Note that $\left|R e \mu_{j}\right| \geq \gamma_{3}$ for any $j \in \boldsymbol{N}$ by Lemma 7.1. Representing each $\boldsymbol{b}_{n}(r)$ as $\boldsymbol{b}_{n}(r)=\sum_{j=1}^{N} \beta_{j}(r) \boldsymbol{e}_{j}$ and substituting into (7.8), we have

$$
\beta_{j}^{\prime \prime}+\frac{1}{r} \beta_{j}^{\prime}-\frac{n^{2}}{r^{2}} \beta_{j}-\mu_{j}^{2} \beta_{j}=0
$$

for each $\boldsymbol{b}_{n}$. Solutions of (7.9) are given by the Bessel functions $Z_{n}\left(\mu_{j} r\right)$, where $Z_{n}(s)$ is a solution of modified Bessel's differential equation

$$
Z_{n}^{\prime \prime}+\frac{1}{s} Z_{n}^{\prime}-\left(1+\frac{n^{2}}{s^{2}}\right) Z_{n}=0 .
$$

Since $Z_{n}(s)$ has an asymptotic profile $O\left(\frac{1}{\sqrt{s}} e^{ \pm s}\right)$ and $\left|\operatorname{Re} \mu_{j}\right| \geq \gamma_{3}, \beta_{j}(r)$ also has an asymptotic profile $O\left(\frac{1}{\sqrt{r}} e^{ \pm c r}\right)$ for $c>0$. Therefore, $\left|\beta_{j}(r)\right| \leq$ 
$O\left(e^{-c^{\prime} / \sqrt{\delta}}\right) \mid \beta_{j}\left(r_{1} / \sqrt{\delta}\right)$ in $r \in\left[0, r_{0}\right]$ for $c^{\prime}>0$, and consequently we have

$$
\|V(r, \theta)\|_{C^{2}\left(B_{0}^{*}\right)} \leq O\left(e^{-c^{\prime} / \sqrt{\delta}}\right)\left\|V\left(r_{1} / \sqrt{\delta}, \theta\right)\right\|_{\infty} \leq O\left(e^{-c^{\prime} / \sqrt{\delta}}\right)\|V\|_{C^{0}\left(B_{1}^{*}\right)} .
$$

This concludes the proof of (7.3).

\subsection{Proof of (4.4)}

In this subsection, we construct the map $\Pi(h): E^{\perp}\left(h_{0}\right) \rightarrow E^{\perp}(h)$ satisfying (4.4).

When we consider $L(h)$ in the domain $\Omega_{1}^{\prime}:=\Omega \backslash \Omega_{0}, L(h)$ is written as

$$
L(h)=\delta^{2} \Delta+F^{\prime}(S(r / \delta))
$$

for $0 \leq s \leq s_{0}$ and $0 \leq z \leq N_{1} \sqrt{\delta}$, where $r=r(\boldsymbol{x} ; h)$. Let $U \in E^{\perp}\left(h_{0}\right) . U$ is expressed as $U=U(s, z)$ in $\bar{\Omega}_{1}^{\prime}$. Hence we define the map $\widehat{\Pi}(h)$ by

$$
(\widehat{\Pi}(h) U)(s, z)=U(s-h, z)
$$

in $\Omega_{1}^{\prime}$, and define in $\Omega_{0}$

$$
(\widehat{\Pi}(h) U)(\boldsymbol{x})=U(\boldsymbol{x})+V(h)(\boldsymbol{x}),
$$

where $V=V(h)(\boldsymbol{x})$ is a function satisfying

$$
L_{0} V=0, x \in \Omega_{0}, \quad V=U(s-h, z)-U(s, z), x \in \partial \Omega_{0}
$$

Note that the function $V$ satisfying (7.11) is uniquely determined because of the invertibility of $L_{0}$. Finally, we give the map $\Pi(h): E^{\perp}\left(h_{0}\right) \rightarrow E^{\perp}(h)$ by $\Pi(h):=$ $R(h) \widehat{\Pi}(h)$. By this construction, (4.4) obviously holds.

\section{References}

1. Alikakos, N.D., Bates, P., Chen, X., Fusco, G.: Mullins-Sekerka motion of small droplets on a fixed boundary. J. Geom. Anal. 10, 575-596 (2000)

2. Alikakos, N.D., Chen, X., Fusco, G.: Motion of a droplet by surface tension along the boundary. Calc. Var. 11, 233-305 (2000)

3. Alikakos, N.D., Fusco, G.: Slow dynamics for the Cahn-Hilliard equation in higher space dimensions. The motion of bubbles. Arch. Ration. Mech. Anal. 141, 1-61 (1998)

4. Alikakos, N.D., Fusco, G., Karali, G.: Motion of bubbles towards the boundary for the Cahn-Hilliard equation. Eur. J. Appl. Math. 15(1), 103-124 (2004)

5. Bates, P.W., Lu, K., Zeng, C.: Approximately invariant manifolds and global dynamics of spike states. Invent. Math. 174, 355-433 (2008)

6. Ei, S.-I.: The motion of weakly interacting pulses in reaction-diffusion systems. J. Dyn. Differ. Equ. 14(1), 85-137 (2002)

7. Ei, S.-I., Ikeda, K., Miyamoto, Y.: Dynamics of a boundary spike for the shadow Gierer-Meinhardt system. Commun. Pure Appl. Anal. 11(1), 115-145 (2012) 
8. Ei, S.-I., Wei, J.: Dynamics of metastable localized patterns and its application to the interaction of spike solutions for the Gierer-Meinhardt systems in two spatial dimension. Jpn. J. Indust. Appl. Math. 19(2), 181-226 (2002)

9. Gierer, A., Meinhardt, H.: A theory of biological pattern formation. Kybernetik (Berlin) 12, 30-39 (1972)

10. Gui, C., Wei, J.: On multiple mixed interior and boundary peak solutions for some singularly perturbed Neumann problems. Can. J. Math. 52(3), 522-538 (2000)

11. Miyamoto, Y.: On the shape of the stable patterns for activator-inhibitor systems in two-dimensional domains. Q. Appl. Math. 65, 357-374 (2007)

12. Miyamoto, Y.: Stability of a boundary spike layer for the Gierer-Meinhardt system. Eur. J. Appl. Math. 16(4), 467-491 (2005)

13. Ni, W.-M., Takagi, I.: Locating the peaks of least-energy solutions to a semilinear Neumann problem. Duke Math. J. 70(2), 247-281 (1993)

14. Turing, A.M.: The chemical basis of morphogenesis. Philos. Trans. Roy. Soc. Lond. Ser. B 237, 37-72 (1952)

15. Wei, J.: Pattern formations in two-dimensional Gray-Scott model: existence of single-spot solutions and their stability. Physica D 148, 20-48 (2001)

16. Wei, J., Winter, M.: Multiple boundary spike solutions for a wide class of singular perturbation problems. J. Lond. Math. Soc. 59, 585-606 (1999) 